Research Article

\title{
Comparing Adolescent Only Children with Those Who Have Siblings on Academic Related Outcomes and Psychosocial Adjustment
}

\author{
Zeng-yin Chen ${ }^{1}$ and Ruth X. Liu ${ }^{2}$ \\ ${ }^{1}$ Department of Sociology, California State University, San Bernardino, 5500 University Parkway, San Bernardino, CA 92407, USA \\ ${ }^{2}$ Department of Sociology, San Diego State University, 5500 Campanile Drive, San Diego, CA 92182-4423, USA \\ Correspondence should be addressed to Zeng-yin Chen; zchen@csusb.edu
}

Received 27 September 2013; Revised 8 December 2013; Accepted 9 December 2013; Published 14 January 2014

Academic Editor: Ross Flom

Copyright (C) 2014 Z.-y. Chen and R. X. Liu. This is an open access article distributed under the Creative Commons Attribution License, which permits unrestricted use, distribution, and reproduction in any medium, provided the original work is properly cited.

\begin{abstract}
This study uses a large and representative sample of adolescents to test the theoretically informed hypotheses comparing adolescent singletons with those who have siblings. The results found that, for academic related outcomes (educational expectations, time spent on homework, and self-reported grades), there are no differences between singletons and firstborns who have any number of younger siblings. Singletons are also not different from laterborns from two-child families. In contrast, singletons are more advantageous compared to laterborns who have two or more siblings on educational expectations and grades. Singletons also spend more time on homework than laterborns who have three or more siblings. For psychosocial outcomes (psychological distress, susceptibility to negative peer pressure, and problem behaviors), singletons are not different from both firstborns and laterborns with any number of siblings. The findings suggest that singletons are not at any disadvantage compared to their peers who have siblings and they enjoy some advantages over laterborns from medium to large families on academic related outcomes.
\end{abstract}

\section{Introduction}

It is a common belief that siblings provide a necessary social environment for a child's normal development. Recent surveys based on nationally representative samples of the US population indicate that only about $3-3.5 \%$ of respondents replied that one child would be the ideal number of children for a family $[1,2]$. However, the US Census of 2008 showed that $20 \%$ of American families had only one child [3]. With a societal trend towards increasingly smaller families, the proportion of singletons (i.e., only children) has been growing in America. This is partly due to people getting married and having children at a later age, along with more serious concerns for family's financial situations [2]. Therefore, the well-being of singletons deserves due attention. Our study thus addresses the concerns that whether singletons, with their rising number, can do well in their academic tasks to get prepared for future careers and whether they can grow into healthy and responsible citizens.
There are two views in the existing literature regarding the welfare of singletons compared to those who have siblings. One view, originally proposed by early psychiatrists and psychologists $[4,5]$ and consistent with the popular thinking $[6,7]$, sees only children as relatively problematic and disadvantaged. With only one child, parents are likely to yield to the only child's demands. Overindulgence is more likely to characterize the parenting style for singletons and thus more likely to mold them into self-centered, dependent, temperamental, and maladjusted individuals $[2,6,8,9]$. Singletons are also expected to be deficient in social skills because they do not have siblings to interact with. Early psychologists even called singletons as a "disease in itself" (G. Standley Hall, as cited in [5]: 547) and society would be better off if "there are no only children" [4]. Similar concerns have been voiced in Chinese literature that China's "onechild" state policy, in effect since 1979 to combat population explosion, is likely to produce a large number of social misfits [10-14]. This unflattering image has influenced the perception 
of psychology counselors, who tend to have preconceptions of only children as more likely to have problems [15].

Another perspective, more based on empirical research, views the single-child status in more positive terms. Studies to date have not provided support for the unfavorable depictions of only children (see updated review by [16]). The results from the meta-analyses of research conducted in the Western settings have reported that only-children are either about the same or more advantageous than children who have siblings [17-19]. Compared to children from medium and large families, singletons have reportedly scored higher in school performance and had higher achievement motivation, higher self-esteem, and higher abilities, especially in verbal skills. Studies have not found significant differences between only children and those who have siblings in the domains of adjustment and sociability $[16,17]$. Singletons are more prevalent in China due to the Chinese government's enforcement of one-child policy. Studies of only children in China have reported that they do better than those who have siblings in academic performance $[13,20,21]$, educational expectations, association with conventional peers, and psychological and behavioral adjustments [22].

Yet empirical findings to date are by no means in consensus regarding the well-being of singletons. Even the relatively more consistent pattern for only children's better academic performance has variations. Some studies based on Western data reported that only children either showed no difference or even scored slightly lower on test scores or educational attainment than their counterparts from small families (i.e., two-child families) $[23,24]$. The findings comparing singletons and those with siblings on academic achievement seem to vary by age too. Among preadolescents, singletons have been reported to perform better academically than those with siblings. Among adolescents, however, there are apparently no differences between singletons and those who have one sibling, although singletons still have higher scores than those who have multiple siblings (see review by [16]). A study on singletons in China has not found significant differences between singletons and nonsingletons in their self-reported school performance [22].

Studies focusing on personality traits and psychological characteristics have also provided mixed findings [16]. Whereas some studies have reported no differences between singletons and those who have siblings $[13,16]$, some have reported more positive outcomes for singletons [20, 22], and still others have presented less favorable outcomes $[25,26]$. Using a clinical sample of disrupted families in therapy, Weiss [27] has observed that only children from divorced families are significantly worse off compared to those who have siblings. Polit's study [28], however, has used a nonclinical sample and found only children from divorced families are doing better in a number of psychological outcomes than those with siblings. Based on a nationally representative sample of kindergarteners, Downey and Condron [29] have reported that children who have siblings are doing better than only children in forming and maintaining friendships. Bobbitt-Zeher and Downey's study [30], however, has used a nationally representative sample of adolescents and reported no difference between singletons and those who have siblings in social skills measured by peer nominations regarding friendship.

The mixed findings on only children from the existing research are partly due to limitations and methodological differences including sample size, measurement, and source of data $[13,16]$. What is more, studies on only children have mostly been data driven searching for differences instead of being guided by theories. There has been some development of theoretical perspectives, which, however, have been used as post hoc explanations of the results [17] rather than guiding theoretical frameworks.

In an attempt to further clarify the existent findings with theoretical guidance, our study uses a large and representative sample of high school students to test our theoretically informed hypotheses regarding the advantages/disadvantages of adolescent singletons in comparison with those who have different number of siblings. We also compare singletons with firstborns and laterborns, respectively, to permit for birth order effects. Two domains of outcomes that parents of only children may be most concerned of will be the focus of our study. (1) Academic domain: how do only children compare to those who have siblings in school related outcomes? (2) Psychosocial domain: how do only children compare to those who have siblings in healthy growth and responsible behavior? This second domain is especially important. Adolescence is a stage of human development that is apparently more capricious than any other stages. Past studies have noted that psychological and behavioral problems (such as mood disruptions and risk behaviors) tend to occur more frequently during adolescence than any other stages of the life cycle [31].

\section{Theoretical Framework}

Our study is theoretically informed by the confluence model, the resource dilution model, and attachment theory. These three theoretical perspectives focus on the influence of the family size on children's outcomes. Although they argue for the same causal relationship, the mechanisms proposed in these three theories vary.

2.1. The Confluence Model. The confluence model attempts to explain the relationship between family size and children's intelligence [32]. The model focuses on the aspect of family intellectual environment and argues that children's environment is primarily defined by the family they live in. Thus, children's family experience has the most important influence on their intelligence. The model's name refers to the phenomenon that various levels of intellectual maturity in a family (such as maturity of parents and that of children at different age) are flowing together and influencing each other; the average level of intellectual maturity keeps changing, especially when a new born joins the family [33]. The level of intelligence in the family stands for the average of combined intelligence of all members without controlling for age. Thus singletons or firstborns mainly interact with adults, which is presumably more stimulating for intellectual growth. As each new child is born to the family, the level of intelligence 
in the family is lowered to accommodate the younger one. The laterborn children not only interact with parents but also with older siblings, whose maturity levels depend on their age. Various predictions of the confluence model have been supported by later studies [33-35]. Thus the confluence model explains the advantages of only children's academic advancement in terms of their interactions primarily with adults in the family, which facilitates their intellectual growth.

2.2. The Resource Dilution Model. Similar to the confluence model but simpler in explanation, the resource dilution model also presents an argument regarding the relationship between family size and children's outcomes [36]. Researchers have long been looking for influential factors on educational attainment. The number of siblings at home has been reported to be inversely related to educational attainment $[24,37,38]$. The resource dilution model offers a simple explanation for this relationship [36]. Starting with an assumption that family resources are finite, the resource dilution model argues that each child that joins the family will dilute the family resources and make every other child in the family receive a smaller share. Blake [36] describes three types of finite family resources: (1) settings, which refer to "types of homes, necessities of life, and cultural objects (like books, pictures, music, and so on;)" (2) opportunities, which include "specific chances to engage the outside world;" and (3) treatments, which include "personal attention, intervention, and teaching" ([36], p. 422). The resource dilution model asserts that these resources are divided by the number of children in a family and the quality of children's lives drops as the family expands. It is further tested that some resources, such as economic ones, are more finite than others and will drop more rapidly as the number of children increases. Interpersonal resources, such as parental attention, have a relatively less severe decline since it is relatively feasible for parents to stretch their attention and energy than economic resources [39].

The causal reasoning regarding the relationship between family size and children's outcomes can be readily applied to the advantages of the singleton status comparing to those with siblings. Unlike children with siblings who have to compete for the scarce financial resources as well as parental attention, singletons have these undivided properties, which are supposed to contribute to their education opportunities, their physical health, and their general well-being [7, 17]. Singletons' parents may also have higher expectations for their only child than parents of multiple children. A heightened parental expectation can be instrumental for the development of achievement motivation $[7,11]$.

In line with the resource dilution model, Hertwig et al. [40] address the parental investment in raising children. The concept of parental investment refers to the cost that parents make in raising a child, which, as a result, will diminish parents' ability to invest in other children [41]. Hertwig and his associates propose three general categories of parental resources that can be invested: (1) material resources, which represent financial wealth and anything that can be obtained with money; (2) cognitive resources, which refer to the family's intellectual environment including the time used in teaching children; and (3) interpersonal resources, which encompass parental attention, love, encouragement, time, and so forth. Thus any parental resources invested in the early-born will reduce the availability of the resources for the laterborn. This prediction is in accordance with the advantage of singletons, whose well-being is maximized because parents are able to invest fully in their only children.

2.3. Attachment Theory. Attachment literature also informs our theoretical reasoning regarding singletons' development. Attachment theory is originally based on the parent-infant relationship, with a basic premise that the primary goal of an infant attachment system is to obtain and keep the caregiver at the time of need. Caregiver's availability and responsiveness tend to result in a felt security in infants, which is a fundamental feeling of the environment as safe, others as loving, and oneself as valuable [42]. With repeated interactions with the attachment figures/caregivers, children are likely to develop an internal working model or representation about attachment, which is to be incorporated into the personality and forms a prototype to guide future relationships [43, 44]. Subsequent research has confirmed the importance of the attachment relationship beyond infancy. Attachment bonds with parents during adolescence continue to provide a solid basis for adolescents' psychological well-being $[45,46]$.

Attachment theory's focus on the early relationship with caregivers lends support for the argument on singleton's well-being. Parents of only children and firstborns have reportedly been more anxious and tense in childcare at their first experience of parenthood [47]. At the laterborns, childcare gets easier with experience and parents become increasingly calmer. The higher level of parental anxiousness of only children leads to their greater extent of concern and responsiveness to their children's needs [7]. Whereas popular thinking tends to see this kind of attentive and responsive parenting as indulgence and overprotection with unwanted outcomes, it is regarded as desirable by attachment theory for providing children with a sense of security that is fundamental for their healthy development. Therefore, greater parental attention devoted to only children is expected to be instrumental in facilitating their development of intellectual competence, psychological confidence, and mature social behaviors.

In sum, the confluence model informs us that singletons will benefit from a higher proportion of interactions with adults at home for their intellectual development. The resource dilution model and attachment theory inform us that the undivided economic resources coupled with undivided and heightened parental availability, attentiveness, and responsiveness will benefit singletons more than those who have siblings both in academic development and in overall healthy development. Thus,

Hypothesis 1. Singletons will fare better than those who have siblings in academic related outcomes.

Hypothesis 2. Singletons will fare better than those who have siblings in psychosocial adjustment. 
In comparing singletons with nonsingletons, it is essential to consider birth-order effects because firstborns are also only children in the family until younger siblings arrive. Birth order studies have consistently observed a negative relationship between birth order and intellectual performance; that is, firstborns tend to perform better than secondborns, secondborns are likely to do better than thirdborns, and so forth $[48,49]$. Birth order causally influencing intelligence has been hotly debated for decades and is "replete with contradictory findings and long-standing conceptual disagreements" ([50], p. 1711). The confluence model $[32,51,52]$ and the resource dilution model $[24,36]$ suggest that firstborns have higher cognitive development than laterborns because the former, like singletons, have the benefits of interacting with adults only and enjoying undivided family resources as well as parental attention during their early years, which are presumably more important for their cognitive development. Each laterborn's arrival will dilute the existing mental maturity level and other resources in the family, resulting in a less optimal environment for intellectual growth. In contrast, competing explanations such as admixture hypothesis [5356] arguing that the findings of the relationship between birth order and intelligence are a methodological artifact due to using between-family data to test within-family hypotheses. The relationship between birth order and intelligence is claimed to be spurious due to socioeconomic status, family size, maternal age, parenting style, macro levels of neighborhood, and school effects [54].

Some empirical studies have reported that singletons are not different from firstborns in intellectual performance $[17$, 20]. However, there have also been inconsistent reports about firstborns in younger age doing less well than laterborns, which has prompted the confluence model to add a teaching function [57]. The revised confluence model explains that firstborns in their early years may lag behind laterborns in intellectual performance. Around 11 years of age, however, firstborns will start to outperform laterborns due to the benefits of their tutoring younger siblings. It is posited that tutoring will boost intelligence of the tutor rather than the tutee. Thus, firstborns' intellectual performance not only surpasses that of laterborns but also of singletons, the latter never having an opportunity to tutor younger siblings and thus at a disadvantage in cognitive development. Since the age group in our study is adolescence, the confluence model would expect only children to do less well than firstborns in academic related outcomes. We will perform additional analyses comparing singletons with firstborns and laterborns to clarify the potentially confounding birth order effects.

\section{Method}

3.1. Sample. Our study uses the survey data collected from approximately 10,000 adolescents from six high schools in northern California and three high schools in Wisconsin during 1987-1988 school year. All of the students from 9th through 12th grades were targeted in the survey. About 15\% of the students were absent on the survey dates and about $5 \%$ refused to participate. Approximately $80 \%$ of the students returned usable questionnaires. For the total sample, $46.1 \%$ of the students were males and $53.9 \%$ were females. Threefourth of the students $(75.6 \%)$ were from California and $24.4 \%$ from Wisconsin. There were $10.2 \%$ from an urban area, $76.9 \%$ from suburban areas, $3.1 \%$ from a farm area, and 9.8\% from an inner city of a metropolitan area. Four ethnic groups yielded sufficient cases for analysis: $66.7 \%$ were non-Hispanic whites, $7.1 \%$ were African Americans, $10.8 \%$ were Hispanics, $13.7 \%$ were Asians, and $1.6 \%$ belonged to other ethnic groups. For parental education, $53.2 \%$ of the respondents reported fathers with four-year college education or beyond and $40.3 \%$ reported mothers with this level of education. Nearly twothirds (65.6\%) of the respondents lived with two biological parents, whereas about one-third (34.4\%) had other types of family arrangement. After deleting the cases with missing data for the study variables, the remaining sample for the analyses in this study ranges from 6,330 to 9,079 .

\subsection{Measurement}

3.2.1. Control Variables. Control variables include sociodemographic information of gender, ethnicity, and parental education. To obviate the possible spurious effect of family disruption on the singleton status and outcome variables [23], two-parent family structure is also controlled. Ethnicity is recoded into dummy variables for major ethnic groups of African American, Asian American, and Hispanic American, with non-Hispanic white as the reference category. Parental education takes the average of the highest level of education obtained by the two biological parents of the respondent. If the information is reported for only one parent, the measure takes on the value for the available parent. Family structure is dichotomized, with two-biological-parent families coded as "1" and all other families coded as " 0 ."

3.2.2. Independent Variables. Two measures of sibling sizes, which ask respondents the number of older and younger siblings, respectively, are recoded into dummy variables based on different sibling sizes. There is a dummy variable of singleton status and four dummy variables for all nonsingletons differentiating those who have one, two, three, and four or more siblings, respectively, without controlling for birth order. Next, there are four dummy variables for firstborns who have one, two, three, and four or more younger siblings. Finally, four additional dummy variables of laterborns are recoded to reflect laterborns with one, two, three, and four or more siblings, respectively.

3.2.3. Dependent Variables. Three variables are selected to reflect academic outcomes. Educational expectations is a single item that asks: "considering your situation, what is the highest level that you really expect to go in school?" Respondents choose among six levels: $1=$ leave school as soon as possible; 2 = finish high school; $3=$ get some vocational or college training; $4=$ finish a two-year community college degree; 5 = finish college with a four-year college degree; $6=$ finish college and take further training. Self-reported grades is a four-item index that asks the respondents the average 
grade they receive for each of the following four courses: math, English, science, and social science, with the response categories of A, B, C, D, and F (Alpha = .77). The question about homework is a two-item index that asks the amount of time the respondents currently put into homework each week for the two main classes: math and English (Alpha = .79). Previous research has supported validity of these three indicators $[58,59]$.

Three other variables tap psychosocial adjustment. Psychological distress is a six-item index derived from the Depression Scale of the Center for Epidemiologic Studies (CES-D) [60]. These items ask how often in a month one feels nervous or worried, "low," depressed, tense, irritable, alone, overtired, having trouble sleeping, losing appetite, or eating too much. The four response categories are $1=$ never, $2=$ once, $3=$ twice, and $4=$ three times or more (Alpha $=.84)$. Susceptibility to negative peer pressure is a five-item index: (a) "your friends want you to have a party this weekend because your parents won't be home;" (b) "while shopping your friends decide to take some clothing without paying for it;" (c) "your friends decide to study from a test answer sheet that was found before the test;" (d) "you and your friends find a car with keys in the door and they decide to take it for a ride;" (e) "your friends decide to buy fake IDs from someone." The four response categories for complying with pressure from friends range from $1=$ "definitely would not" to $4=$ "definitely would" (Alpha $=.75$ ). Problem behaviors is a seven-item index: how often one ran away from home, took something of value from another person, got in trouble with the police, got into a physical fight at school, carried a weapon to school, and purposely damaged school property. The response categories are: $1=$ never, $2=$ once or twice, $3=$ several times, and $4=$ often (Alpha $=.82)$. Previous research has supported validity of these three indicators [59].

3.2.4. Analyses. Multiple regression is performed in our statistical analyses. For each of the six dependent variables there are two different models that control for the same sociodemographic variables of gender, ethnicity, parental education, and two-parent family structure. Model 1 takes sibling size into consideration and enters four dummy variables representing nonsingletons with one, two, three, and four or more siblings, respectively. Model 2 takes both sibling size and birth order into account by entering four dummy variables for firstborns and another four dummy variables for laterborns to reflect their having one, two, three, and four or more siblings, respectively. For both models, singleton status serves as a reference category.

\section{Results}

Table 1 shows the frequency and percentage distribution for singletons, nonsingletons, firstborns, and laterborns by sibling sizes. Eight percent of the total sample was singletons, higher than the percentage reflected in the national surveys on desiring one child only in a family $[1,2]$. Approximately one-third of nonsingletons were firstborns and the other twothirds laterborns.
TABLE 1: Frequency and percentage distribution of singletons, nonsingletons, firstborn, and laterborns by sibling size.

\begin{tabular}{lcc}
\hline & Frequency & Percent \\
\hline Total sample & & \\
Singleton & 780 & $8 \%$ \\
Nonsingleton with 1 sibling & 3,267 & $33 \%$ \\
Nonsingleton with 2 siblings & 2,548 & $25 \%$ \\
Nonsingleton with 3 siblings & 1,509 & $15 \%$ \\
$\quad$ Nonsingleton with 4+ siblings & 1,919 & $19 \%$ \\
\hline Total & 10,023 & $100 \%$ \\
\hline Firstborn & & \\
Firstborn with 1 younger sibling & 1,689 & $55 \%$ \\
Firstborn with 2 younger siblings & 848 & $28 \%$ \\
Firstborn with 3 younger siblings & 327 & $11 \%$ \\
Firstborn with 4+ younger siblings & 210 & $7 \%$ \\
\hline Total & 3,074 & $100 \%$ \\
\hline Laterborn & & \\
Laterborn with 1 sibling & 1,578 & $26 \%$ \\
Laterborn with 2 siblings & 1,700 & $28 \%$ \\
Laterborn with 3 siblings & 1,182 & $19 \%$ \\
Laterborn with 4+ siblings & 1,709 & $28 \%$ \\
\hline Total & 6,169 & $100 \%$ \\
\hline
\end{tabular}

Table 2 presents both unstandardized and standardized regression coefficients for three academic related outcomes. For educational expectations, Model 1 shows that nonsingletons who had one or two siblings were not different from singletons. Those who had three or more siblings reported lower educational expectations than singletons did. However, Model 2, which further differentiates nonsingletons into firstborns and laterborns with different number of siblings, reveals what really accounts for the effects of nonsingletons. Firstborns with any number of siblings were not different from singletons on educational expectations. Similarly, laterborns with only one sibling did not differ from singletons either. Laterborns with two or more siblings, however, reported lower educational expectations than singletons.

For the outcome variable of homework, Model 1 shows that nonsingletons who had one or two siblings were not different from singletons in the homework time spent per week. Those who had three or more siblings reported shorter time spent in doing homework than singletons did. Model 2 , however, again demonstrates firstborns with any number of younger siblings were not different from singletons on homework time. Laterborns with one or two siblings did not differ from singletons either. The only significant effects are from laterborns with three or more siblings, who reported spending less time than singletons on homework.

For self-reported grades, the pattern is again similar. Model 1 shows that adolescents who had one or two siblings were not different from singletons. Those who had three or more siblings reported lower grades than singletons. In contrast, Model 2 reveals from whom these significant effects are found. There are no differences between singletons 
TABLE 2: Academic related outcomes: unstandardized and standardized regression coefficients on educational expectations, homework time, and grades-comparing adolescents who have siblings with singletons (reference category).

\begin{tabular}{|c|c|c|c|c|c|c|c|c|c|c|c|c|}
\hline & \multicolumn{4}{|c|}{ Edu. expectations } & \multicolumn{4}{|c|}{ Homework } & \multicolumn{4}{|c|}{ Grades } \\
\hline & \multicolumn{2}{|c|}{ Model 1} & \multicolumn{2}{|c|}{ Model 2} & \multicolumn{2}{|c|}{ Model 1} & \multicolumn{2}{|c|}{ Model 2} & \multicolumn{2}{|c|}{ Model 1} & \multicolumn{2}{|c|}{ Model 2} \\
\hline & Unstan. & Stan. & Unstan. & Stan. & Unstan. & Stan. & Unstan. & Stan. & Unstan. & Stan. & Unstan. & Stan. \\
\hline Female & $0.15^{* * *}$ & 0.08 & $0.15^{* * *}$ & 0.08 & $0.34^{* * *}$ & 0.13 & $0.34^{* * *}$ & 0.13 & $0.15^{* * *}$ & 0.09 & $0.15^{* * *}$ & 0.09 \\
\hline Asian & $0.24^{* * *}$ & 0.08 & $0.24^{* * *}$ & 0.08 & $0.36^{* * *}$ & 0.10 & $0.35^{* * *}$ & 0.10 & $0.34^{* * *}$ & 0.14 & $0.34^{* * *}$ & 0.14 \\
\hline Black & $0.07^{*}$ & 0.02 & 0.06 & 0.02 & $-0.25^{* * *}$ & -0.06 & $-0.25^{* * *}$ & -0.06 & $-0.28^{* * *}$ & -0.10 & $-0.28^{* * *}$ & -0.10 \\
\hline Hispanic & $-0.11^{* * *}$ & -0.04 & $-0.12^{* * *}$ & -0.04 & $-0.14^{* *}$ & -0.04 & $-0.14^{* * *}$ & -0.04 & $-0.15^{* * *}$ & -0.06 & $-0.15^{* * *}$ & -0.06 \\
\hline Parent edu. & $0.19^{* * *}$ & 0.32 & $0.19^{* * *}$ & 0.32 & $0.18^{* * *}$ & 0.23 & $0.18^{* * *}$ & 0.23 & $0.12^{* * *}$ & 0.23 & $0.12^{* * *}$ & 0.23 \\
\hline Two-parent & $0.13^{* * *}$ & 0.07 & $0.13^{* * *}$ & 0.07 & $0.14^{* * *}$ & 0.05 & $0.14^{* * *}$ & 0.05 & $0.19^{* * *}$ & 0.12 & $0.20^{* * *}$ & 0.12 \\
\hline Nonsingleton $1 \mathrm{sib}$ & -0.01 & 0.00 & - & - & -0.01 & 0.00 & - & - & 0.01 & 0.00 & - & - \\
\hline Nonsingleton $2 \mathrm{sib}$ & -0.06 & -0.03 & - & - & -0.07 & -0.02 & - & - & -0.05 & -0.03 & - & - \\
\hline Nonsingleton 3 sib & $-0.10^{*}$ & -0.04 & - & - & $-0.13^{*}$ & -0.04 & - & - & $-0.09^{* *}$ & -0.04 & - & - \\
\hline Nonsingleton $4+$ sib & $-0.14^{* * *}$ & -0.06 & - & - & $-0.15^{*}$ & -0.04 & - & - & $-0.08^{*}$ & -0.04 & - & - \\
\hline Firstborn $1 \mathrm{sib}$ & - & - & -0.01 & 0.00 & - & - & 0.03 & 0.01 & - & - & 0.03 & 0.01 \\
\hline Firstborn 2 sib & - & - & -0.02 & -0.01 & - & - & -0.04 & -0.01 & - & - & 0.00 & 0.00 \\
\hline Firstborn 3 sib & - & - & -0.01 & 0.00 & - & - & -0.04 & -0.01 & - & - & -0.07 & -0.01 \\
\hline Firstborn $4+$ sib & - & - & -0.10 & -0.02 & - & - & -0.03 & 0.00 & - & - & -0.01 & 0.00 \\
\hline Laterborn 1 sib & - & - & -0.01 & 0.00 & - & - & -0.05 & -0.01 & - & - & -0.02 & -0.01 \\
\hline Laterborn $2 \mathrm{sib}$ & - & - & $-0.09^{*}$ & -0.03 & - & - & -0.08 & -0.02 & - & - & $-0.07^{*}$ & -0.04 \\
\hline Laterborn 3 sib & - & - & $-0.13^{* *}$ & -0.04 & - & - & $-0.15^{*}$ & -0.04 & - & - & $-0.10^{* *}$ & -0.04 \\
\hline Laterborn $4+$ sib & - & - & $-0.14^{* * *}$ & -0.05 & - & - & $-0.16^{* *}$ & -0.05 & - & - & $-0.09^{*}$ & -0.04 \\
\hline Constant & 2.77 & & 2.77 & & 2.6 & & 2.61 & & 2.04 & & 2.04 & \\
\hline$R^{2}$ (adj) & 0.14 & & 0.15 & & 0.11 & & 0.11 & & 0.15 & & 0.15 & \\
\hline$N$ & 9,079 & & 9,079 & & 8,355 & & 8,355 & & 8,576 & & 8,576 & \\
\hline
\end{tabular}

${ }^{*} P<0.05 ;{ }^{* *} P<0.01 ;{ }^{* * *} P<0.001$ (two-tailed tests).

and firstborns regardless of the number of siblings firstborns had. Laterborns who had one sibling only were also not different from singletons. Laterborns with two or more siblings, however, reported lower grades than singletons with statistical significance.

As demonstrated in Model 2, firstborns, either from small, medium, or large families, fare as well as singletons in academic related outcomes. In contrast, only laterborns from small families (i.e., two-child families) are comparable with singletons. The laterborns from medium to large families apparently fare less well than singletons in academic related outcomes. The noticeable difference between firstborns and laterborns is consistent with the confluence theory and resource dilution model about birth-order effects on intellectual development.

The effects from control variables on the three academic related outcomes were also worth noting. The result for Model 2 in Table 2 indicated that being a female, having higher parental education, and living with both biological parents were positively related to all three academic related outcomes. They reported higher educational expectations, longer time spent on homework, and getting higher grades. Compared to whites, Asian American students reported higher educational expectations, spending longer time on homework, and obtaining higher grades. African American adolescents reported spending less time on homework, and getting lower grades. Hispanic American adolescents reported lower educational expectations, spending less time on homework, and getting lower grades. Parental education and living in a two-biological-parent household were positively related to educational expectations, time spent on homework, and self-reported grades.

Table 3 reports unstandardized and standardized regression coefficients for psychosocial outcomes. In contrast to the patterns observed for the academic related variables in Table 2, different patterns emerged for psychosocial outcomes in Table 3. After controlling for the sociodemographic background variables, both Model 1 and Model 2 demonstrate the same result. All nonsingletons in Model 1, or firstborns and laterborns regardless of their sibling sizes in Model 2, did not differ from singletons in psychological distress, susceptibility to negative peer pressure, and problem behavior.

Table 3 also presents the effects of control variables on the three psychosocial outcomes. Female adolescents reported more psychological symptoms than males but were less likely to yield to negative peer pressure and had lower levels of problem behavior. Compared to white adolescents, Asian American, African American, and Hispanic American adolescents reported fewer psychological symptoms. Asian American and African American adolescents were less likely to yield to negative peer pressure, and Asian American adolescents also reported lower levels of problem behavior. Parental education was positively related to psychological distress but negatively associated with problem behavior. Living 
TABLE 3: Psychosocial outcomes: unstandardized and standardized regression coefficients psychological symptoms, susceptibility to negative peer pressure, and problem behaviors-comparing adolescents who have siblings with singletons (reference category).

\begin{tabular}{|c|c|c|c|c|c|c|c|c|c|c|c|c|}
\hline & \multicolumn{4}{|c|}{ Psychological distress } & \multicolumn{4}{|c|}{ Susceptibility to neg. peer pressure } & \multicolumn{4}{|c|}{ Deviant behavior } \\
\hline & \multicolumn{2}{|c|}{ Model 1} & \multicolumn{2}{|c|}{ Model 2} & \multicolumn{2}{|c|}{ Model 1} & \multicolumn{2}{|c|}{ Model 2} & \multicolumn{2}{|c|}{ Model 1} & \multicolumn{2}{|c|}{ Model 2} \\
\hline & Unstan. & Stan. & Unstan. & Stan. & Unstan. & Stan. & Unstan. & Stan. & Unstan. & Stan. & Unstan. & Stan. \\
\hline Female & $0.52^{* * *}$ & 0.32 & $0.52^{* * *}$ & 0.32 & $-0.24^{* * *}$ & -0.18 & $-0.24^{* * *}$ & -0.18 & $-0.16^{* * *}$ & -0.22 & $-0.16^{* * *}$ & -0.22 \\
\hline Asian & $-0.06^{*}$ & -0.02 & $-0.06^{*}$ & -0.02 & $-0.26^{* * *}$ & -0.14 & $-0.26^{* * *}$ & -0.14 & $-0.08^{* * *}$ & -0.08 & $-0.08^{* * *}$ & -0.08 \\
\hline Black & $-0.31^{* * *}$ & -0.11 & $-0.30^{* * *}$ & -0.11 & $-0.20^{* * *}$ & -0.09 & $-0.20^{* * *}$ & -0.09 & -0.01 & -0.01 & -0.01 & -0.01 \\
\hline Hispanic & $-0.16^{* * *}$ & -0.06 & $-0.16^{* * *}$ & -0.06 & -0.03 & -0.02 & -0.03 & -0.01 & 0.01 & 0.01 & 0.01 & 0.01 \\
\hline Parent edu. & $0.01^{* *}$ & 0.03 & $0.01^{* *}$ & 0.03 & 0.00 & -0.01 & 0.00 & -0.01 & $-0.01^{* * *}$ & -0.05 & $-0.01^{* * *}$ & -0.05 \\
\hline Two-parent & $-0.08^{* * *}$ & -0.05 & $-0.08^{* * *}$ & -0.05 & $-0.07^{* * *}$ & -0.05 & $-0.07^{* * *}$ & -0.05 & $-0.02^{*}$ & -0.03 & $-0.02^{*}$ & -0.03 \\
\hline Nonsingleton $1 \mathrm{sib}$ & 0.01 & 0.01 & - & - & 0.04 & 0.03 & - & - & 0.02 & 0.03 & - & - \\
\hline Nonsingleton $2 \mathrm{sib}$ & 0.01 & 0.01 & - & - & 0.02 & 0.01 & - & - & 0.02 & 0.02 & - & - \\
\hline Nonsingleton $3 \mathrm{sib}$ & 0.00 & 0.00 & - & - & -0.01 & -0.01 & - & - & -0.01 & -0.01 & - & - \\
\hline Nonsingleton $4+$ sib & -0.01 & -0.01 & - & - & -0.03 & -0.02 & - & - & 0.02 & 0.03 & - & - \\
\hline Firstborn $1 \mathrm{sib}$ & - & - & -0.01 & 0.00 & - & - & 0.04 & 0.02 & - & - & 0.02 & 0.02 \\
\hline Firstborn 2 sib & - & - & 0.00 & 0.00 & - & - & -0.02 & -0.01 & - & - & 0.00 & 0.00 \\
\hline Firstborn 3 sib & - & - & -0.01 & 0.00 & - & - & -0.04 & -0.01 & - & - & -0.02 & -0.01 \\
\hline Firstborn $4+$ sib & - & - & 0.01 & 0.00 & - & - & -0.11 & -0.03 & - & - & -0.01 & 0.00 \\
\hline Laterborn 1 sib & - & - & 0.04 & 0.02 & - & - & 0.05 & 0.03 & - & - & 0.02 & 0.02 \\
\hline Laterborn 2 sib & - & - & 0.01 & 0.01 & - & - & 0.04 & 0.02 & - & - & 0.02 & 0.03 \\
\hline Laterborn 3 sib & - & - & 0.00 & 0.00 & - & - & -0.01 & 0.00 & - & - & -0.01 & -0.01 \\
\hline Laterborn $4+$ sib & - & - & -0.02 & -0.01 & - & - & -0.02 & -0.01 & - & - & 0.03 & 0.03 \\
\hline Constant & 2.28 & & 2.27 & & 2.27 & & 2.27 & & 1.33 & & 1.33 & \\
\hline$R^{2}$ (adj) & 0.12 & & 0.12 & & 0.06 & & 0.06 & & 0.06 & & 0.06 & \\
\hline$N$ & 8,043 & & 8,043 & & 6,490 & & 6,490 & & 6,330 & & 6,330 & \\
\hline
\end{tabular}

${ }^{*} P<0.05 ;{ }^{* *} P<0.01 ;{ }^{* * *} P<0.001$ (two-tailed tests).

with two biological parents predicted lower psychological distress, less susceptibility to negative peer pressure, and lower levels of problem behavior.

\section{Discussion}

Our study attempts to investigate differences between adolescents who have no siblings and those who have different number of siblings in two different domains, academic related outcomes and psychosocial adjustment. Interesting patterns have emerged after controlling for sociodemographic background variables of gender, ethnicity, parental education, and two-parent family structure. For all three academic related outcomes-educational expectations, time spent on homework and grades-there is no significant difference between singletons and firstborns who have any number of younger siblings. Singletons do not differ from laterborns from small families (i.e., two-child families) either but fare better than laterborns from medium to large families (i.e., three-or-more-child families) in reporting higher educational expectations and getting better grades. Singletons also spend more time on homework than laterborns from four-child-families. For psychosocial adjustment, however, a different pattern is observed. Singletons manage about the same as both firstborns and laterborns with any sibling sizes in psychological distress, susceptibility to negative peer pressure, and problem behaviors.

Our results of comparing singletons and nonsingletons on academic related issues are consistent with the confluence model [32, 51], the resource dilution model $[24,36]$, and attachment theory $[43,44]$ and hence support our first hypothesis in general. Our study provides another confirmation to the reports by other studies [16-19] and is also consistent with the literature on the relationship between birth order and intellectual performance [24, 35, 36, 48, 49]. Apparently, family size has a measurable effect on academic outcomes. A family's overall mental maturity level, undivided resources, as well as heightened parental responsiveness and care may all have assisted singletons in their schooling. In addition, the benefits of family resources on academic outcomes also promote firstborns and can be extended to laterborns but not without decrement. The decreasing benefits are shown for laterborns from medium to large families in their reported lower educational expectations, less time spent on homework, and lower grades.

It is important to note that the observed advantages for singletons, firstborns, and those from small families remain in academic related outcomes only. Our second hypothesis that expects singletons' faring better in psychological and behavioral outcomes is not supported because there is largely no difference between singletons and those who have any number of siblings. As a matter of fact, this result is consistent 
with other studies that have failed to find differences between singletons and those who have siblings on adjustment and sociability $[16,17]$. It is reassuring that the draining of family economic and parental resources from medium to large families does not negatively affect children's psychosocial wellbeing. The findings may be regarded as somewhat consistent with the study by Strohschein and her associates [61] that adding more children into the family does not necessarily drain parenting resources. Rather, parental behavior may have gone through readjustment, such as reducing emotional nurturing and time spent with older children on one hand and increasing consistent parenting such as limit setting and ensuring appropriate behavior on the other [61].

Cross-culturally, our findings are consistent with other studies on Chinese singletons [13, 20-22]. Singletons in China grow up in an environment where the Chinese government has implemented the one-child policy throughout the country since 1979 to curb population growth. In contrast, only children in the Western world are not common. Families with singletons in the US may have had some unmeasured disadvantages such as disrupted marital relationship or developmental difficulties of the firstborn [36]. Yet, the outcomes for only children in these two completely different cultural environments are remarkably similar. Falbo and Poston's studies have found that only children in China consistently outperform those who have siblings in school performance and standard tests but they are not different from those siblinged counterparts in personality, whether rated by their peers, parents, teachers, or themselves [20]. Liu and others have reported that Chinese singletons do better in educational expectations, association with conventional peers, and psychological and behavioral adjustments [22]. Our study reinforces a clear message sent by those researchers that singletons are certainly not at a disadvantage. They are no more spoiled than their siblinged counterparts regardless of the societal environments in which they have been raised, whether in China or in the US.

This study contributes to the existing research in debunking the myth on the unfavorable view about singletons. While singletons do not differ from those who have siblings in psychosocial adjustment, they are likely to enjoy some measureable advantages over laterborns from medium to large families in academic related outcomes. Apparently, the myth of singleton status being problematic or disadvantageous does not bear any warrant in our study. Despite the commonly held stereotype about only children being maladjusted and socially undesirable, they actually fare as well as other adolescents during this capricious developmental stage. It is reassuring that singletons are likely to grow into healthy and responsible citizens, same as those who have siblings. What is more, singletons, similar to firstborns and those children from two-child families, are observed with slightly higher levels of academic outcomes. Nevertheless, we may speculate that laterborns who are more intelligent or more dedicated in academics do well in school regardless of family size. It might be those children who are at the average level or those who are struggling in school probably benefit more from concentrated family intellectual properties and financial resources as well as parental availability of one-on-one care and assistance, which may have contributed to a statistical difference in the academic advantages enjoyed by adolescents who are either singletons, firstborns, or from small families.

Our study, however, has not found that singletons perform less well than firstborns on academic outcomes as predicted by the confluence theory. It is possible that singletons' lack of tutoring benefits on intelligence may not be reflected in educational expectations, homework time, and self-reported GPA. Standardized tests, if available, will be a more rigorous measurement of cognitive performance.

We must admit that the coefficients of laterborns from medium to large families on academic outcomes are generally small, which suggest small effect sizes. The small but statistically significant effect sizes could be attributed to a large sample size. Thus, we should be cautious in drawing our conclusions regarding the effect of sibling sizes. The findings merely suggest that there is a very small yet detectable association between sibling sizes and decrement of academic outcomes.

We admit the limitation of using an existing data set that does not have a more adequate measurement of socioeconomic status besides parental education. Although parental education is defended as the most stable indicator of social class and it typically accounts for most of the effects of parental income on children's achievement [62], measures of family financial situation, and parental occupation, if available, it will definitely strengthen the control for the socioeconomic environment of the family. Another shortcoming of our study is a complete reliance on a single source of respondents' self-report, which may have artificially inflated associations among all variables. Data limitations also prevent us to study some other domains that people are concerned of regarding singletons such as temperament, dependency, self-centeredness, and so on.

This study also leads to more paths for further research. It is important to continue studying singletons beyond adolescence and examine how they perform in their adult roles in career and family. Future research should shed light on whether singletons' advantages in academic arena during childhood and adolescence necessarily translate into higher socioeconomic status and more productive careers during adulthood and whether their lack of sibling interaction during their growing up years may influence their marital relationship and their own parenting styles.

\section{Conflict of Interests}

The authors declare that there is no conflict of interests regarding the publication of this paper.

\section{Acknowledgments}

This project was supported by a mini grant award to the first author from California State University, San Bernardino. The study on which this project was based was sponsored by the Spencer Foundation, the Joseph Drown Foundation, and the Office of Educational Research and Improvement with Sanford M. Dornbusch, Laurence Steinberg, and B. Bradford 
Brown as the Principal Investigators. The authors thank Dr. Sanford M. Dornbusch for his permission to use the data set.

\section{References}

[1] "General social survey," The Interuniversity Consortium for Political and Social Research (ICPSR), 2010.

[2] A. Mancillas, "Challenging the stereotypes about only children: a review of the literature and implications for practice," Journal of Counseling and Development, vol. 84, no. 3, pp. 268-275, 2006.

[3] A. Mancillas, "Only children," in Sibling Development: Implications for Mental Health Practitioners, J. Caspi, Ed., pp. 341-358, Springer, New York, NY, US, 2011.

[4] A. A. Brill, Psychoanalysis-Its Theories and Practical Applications, Elsevier Saunders, Philadelphia, Pa, USA, 1922.

[5] N. Fenton, "The only child," Journal of Genetic Psychology, vol. 35, pp. 546-556, 1928.

[6] T. Falbo, "The only-child family in the United States: research issues and results," Studies in Family Planning, vol. 13, no. 6-7, pp. 212-215, 1982.

[7] T. Falbo, "Only children in the United States and China," Applied Social Psychology Annual, vol. 7, pp. 159-183, 1987.

[8] L. C. Roberts and P. W. Blanton, "I always knew mom and dad love me best': experiences of only children," Journal of Individual Psychology, vol. 57, pp. 125-140, 2001.

[9] V. D. Thompson, "Family size: implicit policies and assumed psychological outcomes," Journal of Social Issues, vol. 30, no. 4, pp. 93-124, 1974.

[10] E. N. Chow and S. M. Zhao, "The one-child policy and parentchild relationships: a comparison of one-child with multiplechild families in China," The International Journal of Sociology and Social Policy, vol. 16, pp. 35-62, 1996.

[11] V. A. Fong, Only Hope: Coming of Age under China's One-Child Policy, Stanford University Press, Stanford, Calif, USA, 2004.

[12] E. Hall, "China's only child," Psychology Today, vol. 19, pp. 44-47, 1987.

[13] D. L. Poston and T. Falbo, "Scholastic and personality characteristics of only children and children with siblings in China," International Family Planning Perspectives, vol. 16, no. 2, pp. 45$54,1990$.

[14] K.-T. Tao, "An overview of only child family mental health in China," Psychiatry and Clinical Neurosciences, vol. 52, pp. 206211, 1998.

[15] A. E. Stewart, "Can knowledge of client birth order bias clinical judgment?” Journal of Counseling and Development, vol. 82, no. 2, pp. 167-176, 2004.

[16] T. Falbo, "Only children: an updated review," Journal of Individual Psychology, vol. 68, no. 1, pp. 38-49, 2012.

[17] T. Falbo and D. F. Polit Jr., "Quantitative review of the only child literature: research evidence and theory development," Psychological Bulletin, vol. 100, no. 2, pp. 176-189, 1986.

[18] D. F. Polit and T. Falbo, "Only children and personality development: a quantitative review," Journal of Marriage and the Family, vol. 49, pp. 309-325, 1987.

[19] D. F. Polit and T. Falbo, "The intellectual achievement of only children," Journal of Biosocial Science, vol. 20, no. 3, pp. 275-286, 1988.

[20] T. Falbo and D. L. Poston Jr., "The academic, personality, and physical outcomes of only children in China," Child Development, vol. 64, no. 1, pp. 18-35, 1993.
[21] D. L. Poston and T. Falbo, "Academic performance and personality traits of Chinese children: "Onlies" versus others," American Journal of Sociology, vol. 96, pp. 433-451, 1990.

[22] R. X. Liu, W. Lin, and Z.-Y. Chen, "School performance, peer association, psychological and behavioral adjustments: a comparison between Chinese adolescents with and without siblings," Journal of Adolescence, vol. 33, no. 3, pp. 411-417, 2010.

[23] J. Blake, Family Size and Achievement, University of California Press, Los Angeles, Calif, USA, 1989.

[24] D. B. Downey, "Number of siblings and intellectual development: the resource dilution explanation," American Psychologist, vol. 56, no. 6, pp. 497-504, 2001.

[25] S. Jiao, G. Ji, and Q. Jing, "Comparative study of behavioral qualities of only children and sibling children," Child Development, vol. 57, pp. 357-361, 1986.

[26] C. Liu, T. Munakata, and F. N. Onuoha, "Mental health condition of the only-child: a study of urban and rural high school students in China," Adolescence, vol. 40, no. 160, pp. 831-845, 2005.

[27] R. S. Weiss, Going It Alone, Basic Books, New York, NY, USA, 1979.

[28] D. F. Polit, "The only child in single-parent families," in The Single-Child Family, T. Falbo, Ed., pp. 178-210, Guilford Press, New York, NY, USA, 1984.

[29] D. B. Downey and D. J. Condron, "Playing well with others in kindergarten: the benefit of siblings at home," Journal of Marriage and Family, vol. 66, no. 2, pp. 333-350, 2004.

[30] D. Bobbitt-Zeher and D. B. Downey, "Number of siblings and friendship nominations among adolescents," Journal of Family Issues, vol. 34, pp. 1175-1193, 2013.

[31] J. J. Arnett, "Adolescent storm and stress, reconsidered," American Psychologist, vol. 54, no. 5, pp. 317-326, 1999.

[32] R. B. Zajonc and G. B. Markus, "Birth order and intellectual development," Psychological Review, vol. 82, no. 1, pp. 74-88, 1975.

[33] R. B. Zajonc, "The family dynamics of intellectual development," American Psychologist, vol. 56, no. 6, pp. 490-496, 2001.

[34] R. B. Zajonc, "Validating the confluence model," Psychological Bulletin, vol. 93, no. 3, pp. 457-480, 1983.

[35] R. B. Zajonc and F. J. Sulloway, "The confluence model: birth order as a within-family or between-family dynamic?" Personality and Social Psychology Bulletin, vol. 33, no. 9, pp. 11871194, 2007.

[36] J. Blake, "Family size and the quality of children," Demography, vol. 18, no. 4, pp. 421-442, 1981.

[37] P. M. Blau and O. D. Duncan, The American Occupational Structure, John Wiley \& Sons, New York, NY, USA, 1967.

[38] D. Featherman and R. Hauser, Opportunity and Change, Academic Press, New York, NY, USA, 1978.

[39] D. B. Downey, "When bigger is not better: family size, parental resources, and children's educational performance," American Sociological Review, vol. 60, pp. 747-761, 1995.

[40] R. Hertwig, J. N. Davis, and F. J. Sulloway, "Parental investment: how an equity motive can produce inequality," Psychological Bulletin, vol. 128, no. 5, pp. 728-745, 2002.

[41] R. L. Trivers, "Parent-offspring conflict," American Zoologist, vol. 14, no. 1, pp. 249-264, 1974.

[42] M. J. Cox and K. S. M. Harter, "Parent-child relationships," in Well-Being: Positive Development Across the Life Course, M. H. Bornstein, L. Davidson, C. L. M. Keyes, and K. A. Moore, Eds., pp. 191-204, Lawrence Erlbaum, Mahwah, NJ, USA, 2003. 
[43] J. Bowlby, Attachment and Loss, vol. 1, Basic Books, New York, NY, USA, 2nd edition, 1982.

[44] J. Bowlby, A Secure Base, Routledge, London, UK, 1988.

[45] M. D. S. Ainsworth, "Attachment: retrospect and prospect," in The Place of Attachment in Human Behavior, C. M. Parkes and J. Stevenson-Hinde, Eds., Basic Books, New York, NY, USA, 1982.

[46] G. C. Armsden and M. T. Greenberg, "The inventory of parent and peer attachment: individual differences and their relationship to psychological well-being in adolescence," Journal of Youth and Adolescence, vol. 16, no. 5, pp. 427-454, 1987.

[47] S. Schachter, The Psychology of Affiliation, Stanford University Press, Stanford, Calif, USA, 1959.

[48] L. Belmont and F. A. Marolla, "Birth order, family size, and intelligence. A study of a total population of 19 year old men born in the Netherlands is presented," Science, vol. 182, no. 4117, pp. 1096-1101, 1973.

[49] T. Bjerkedal, P. Kristensen, G. A. Skjeret, and J. I. Brevik, "Intelligence test scores and birth order among young Norwegian men (conscripts) analyzed within and between families," Intelligence, vol. 35, no. 5, pp. 503-514, 2007.

[50] F. J. Sulloway, "Birth order and intelligence," Science, vol. 316, no. 5832, pp. 1711-1712, 2007.

[51] R. B. Zajonc, "Family configuration and intelligence," Science, vol. 192 , no. 4236 , pp. 227-236, 1976.

[52] R. B. Zajonc and P. R. Mullally, "Birth order: reconciling conflicting effects," American Psychologist, vol. 52, no. 7, pp. 685699, 1997.

[53] E. B. Page and G. Grandon, "Family configuration and mental ability: two theories contrasted with U.S. data," American Educational Research Journal, vol. 16, pp. 257-272, 1979.

[54] J. L. Rodgers, "What causes birth order-intelligence patterns? The admixture hypothesis, revived," American Psychologist, vol. 56, no. 6, pp. 505-510, 2001.

[55] J. L. Rodgers, "Are birth order effects on intelligence really flynn effects? Reinterpreting belmont and marolla 40 years later," Intelligence, 2013.

[56] W. Velandia, G. M. Grandon, and E. B. Page, "Family size, birth order, and intelligence in a large South American sample," American Educational Research Journal, vol. 15, no. 3, pp. 399416, 1978.

[57] R. B. Zajonc, H. Markus, and G. B. Markus, "The birth order puzzle," Journal of Personality and Social Psychology, vol. 37, no. 8, pp. 1325-1341, 1979.

[58] Z.-Y. Chen, “The relationship between parental constructive behavior and the adolescent's association with achievement oriented peers: a longitudinal study," Sociological Inquiry, vol. 70, no. 3, pp. 360-381, 2000.

[59] Z.-Y. Chen and S. M. Dornbusch, "Relating aspects of adolescent emotional autonomy to academic achievement and deviant behavior," Journal of Adolescent Research, vol. 13, no. 3, pp. $293-$ 319, 1998.

[60] L. S. Radloff, “The CES-D scale: a self-report depression scale for research in the general population," Applied Psychological Measurement, vol. 1, pp. 385-401, 1977.

[61] L. Strohschein, A. H. Gauthier, R. Campbell, and C. Kleparchuk, "Parenting as a dynamic process: a test of the resource dilution hypothesis," Journal of Marriage and Family, vol. 70, no. 3, pp. 670-683, 2008.

[62] L. Steinberg, N. S. Mounts, S. D. Lamborn, and S. M. Dornbusch, "Authoritative parenting and adolescent adjustment across varied ecological niches," Journal of Research on Adolescence, vol. 1, pp. 19-36, 1991. 

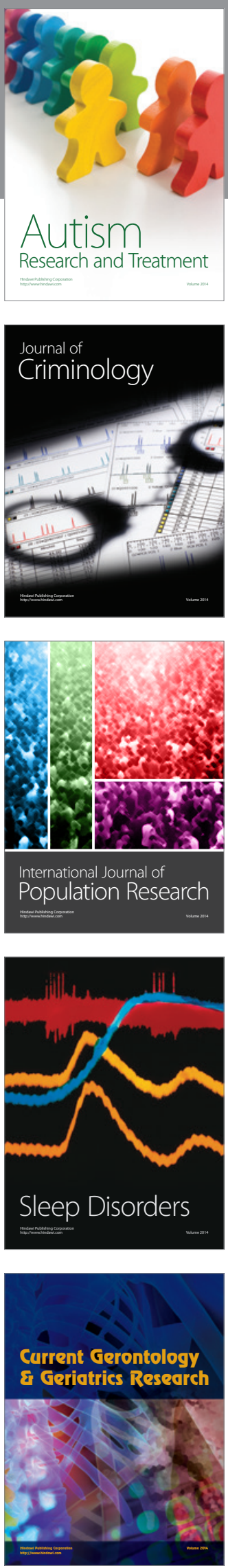
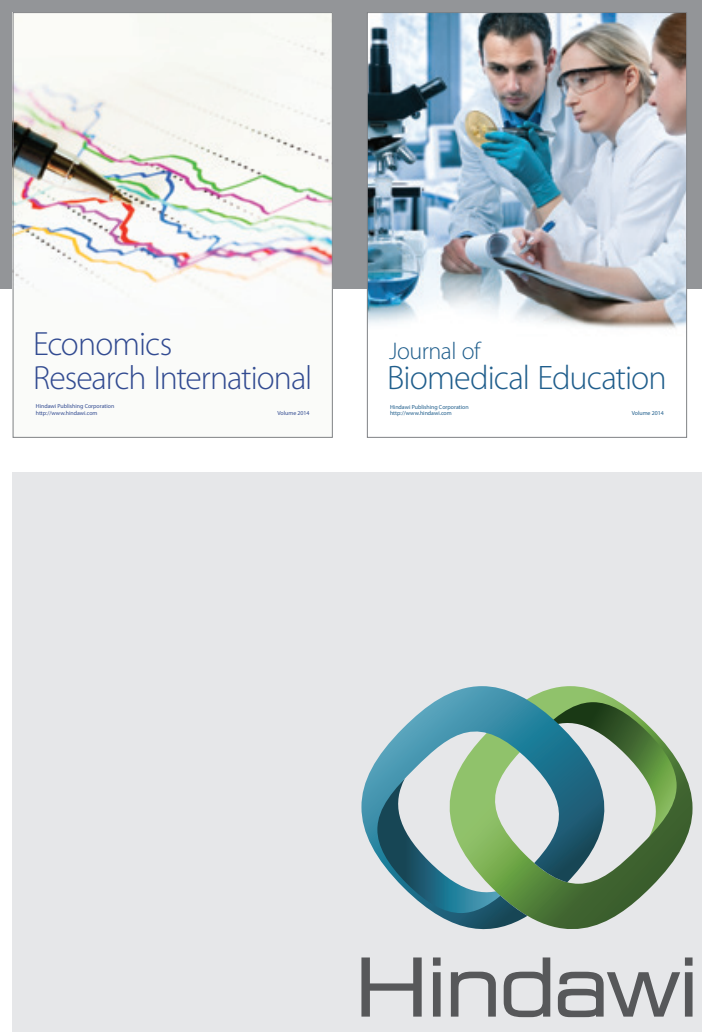

Submit your manuscripts at

http://www.hindawi.com
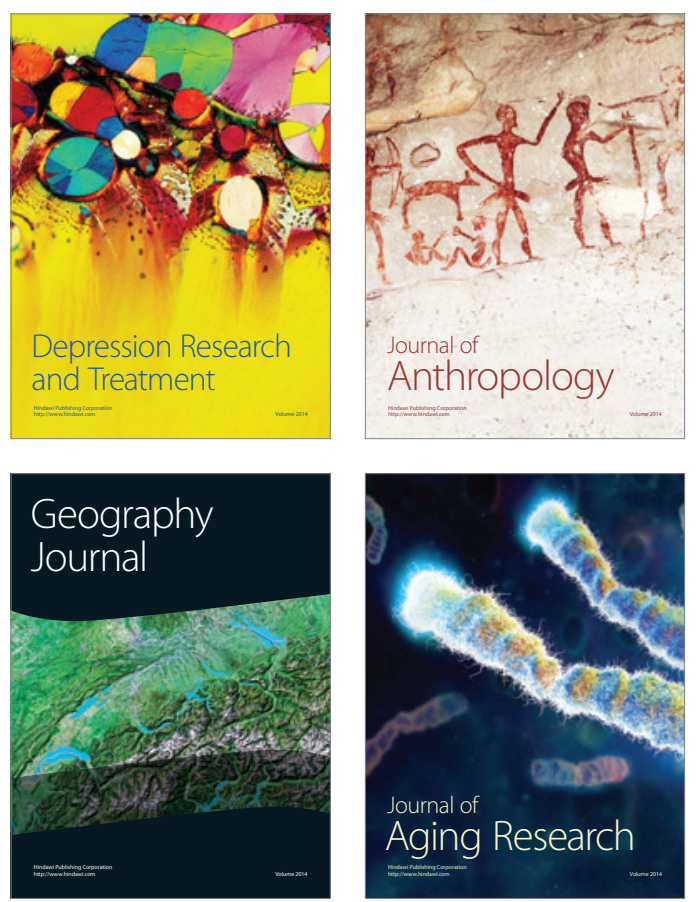
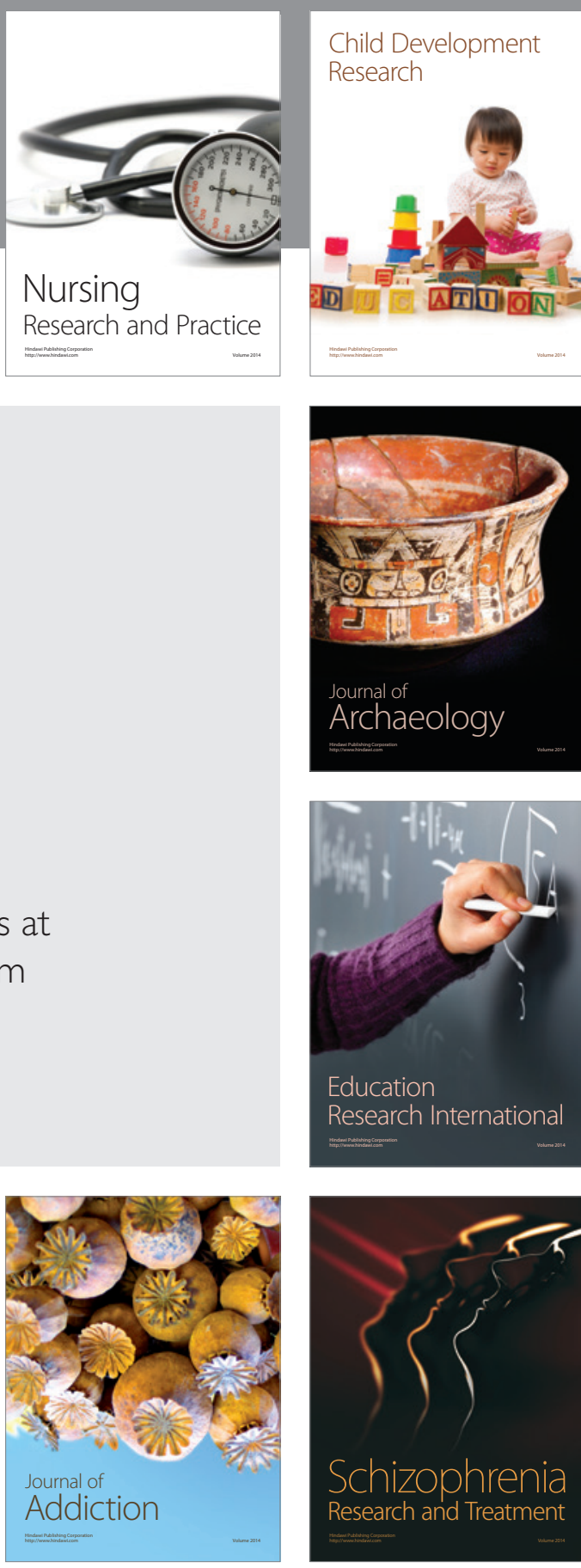

(D)
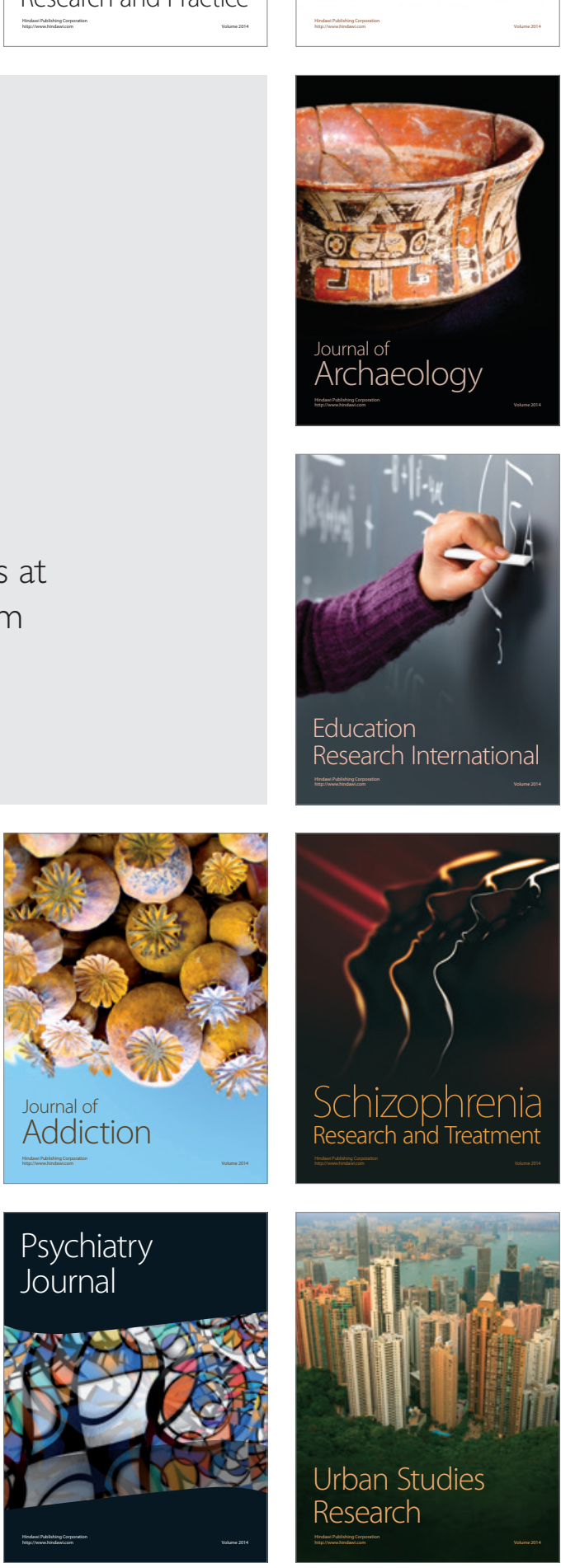